\title{
Necrólisis epidérmica tóxica: reacción idiosincrática a neurofármacos
}

Toxic epidermal necrolysis: idiosyncratic reaction to drugs

\author{
Julio César Rotondaro',Aaron Cristian Kim', Paula Monti', Mónica Pazos³, \\ Carla Gazzi ${ }^{4}$, Rodolfo López ${ }^{5}$, Fernando Riccioppo², Daniel Fadel ${ }^{2}$
}

\section{Resumen}

La necrólisis epidérmica tóxica (NET) y el síndrome de Steven Johnson (SSJ) son reacciones de hipersensibilidad mucocutáneas con afectación sistémica ocasionadas, predominantemente, por fármacos. Se reporta el caso de un paciente mayor de 60 años de edad que presentó lesión mucocutánea extensa y ocular, con fallo hemodinámico, asociado a la rápida instauración de lamotrigina en dosis crecientes en un corto plazo. Si bien la incidencia de esta patología es baja, la tasa de mortalidad es alta, por lo que se requiere de la sospecha y diagnóstico precoces sumado a un abordaje terapéutico interdisciplinario.

Palabras clave: Anticomiciales - Divalproato de sodio - Hipersensibilidad - Lamotrigina - Necrólisis epidérmica tóxica - Síndrome de Steven Johnson.

\begin{abstract}
Toxic epidermal necrolysis (NET) and Steven Johnson syndrome (SJS) are infrequent mucocutaneous hypersensitivity reactions with systemic involvement. They are predominantly caused by drugs. We report the case of a patient over 60 years of age who presented with extensive mucocutaneous and ophthalmic injury with hemodynamic failure, associated with the rapid onset of lamotrigine in a short period of time. Although the incidence is low, the mortality rate is high. It requires early suspicious and diagnosis in addition to an interdisciplinary therapeutic approach.
\end{abstract}

Keywords: Anticonvulsants - Divalproate sodium - Hypersensitivity - Lamotrigine - Necrolysis toxic epidermal - Steven Johnson syndrome.

RECIBIDO II/3/202| - ACEPTADO |4/5/202|

1.Servicio de clínica médica, Instituto de investigaciones médicas Alfredo Lanari.

${ }^{2}$ Servicio de salud mental, Instituto de investigaciones médicas Alfredo Lanari.

3.Servicio de dermatología, Instituto de investigaciones médicas Alfredo Lanari.

4.Servicio de patología, Instituto de investigaciones médicas Alfredo Lanari.

5. Unidad de cuidados intensivos, Instituto de investigaciones médicas Alfredo Lanari.

Autor de referencia:

Julio César Rotondaro

rotondarojuliocesar@gmail.com

Lugar de realización del estudio: Servicio de Clínica Médica, Instituto de investigaciones médicas Alfredo Lanari,

Ciudad Autónoma de Buenos Aires, Argentina. 


\section{Introducción}

La necrólisis epidérmica tóxica (NET) y el síndrome de Steven Johnson (SSJ) son reacciones de hipersensibilidad mucocutáneas con afectación sistémica, predominantemente ocasionadas por fármacos. Constituyen una falla dermo-epidérmica aguda y poco frecuente pero con alta mortalidad, que pueden superar el 30\% en algunos casos (Dodiuk-Gad et al., 2015). Su rápido reconocimiento, el retiro del fármaco responsable y la instauración precoz de medidas de soporte vital son las principales acciones que modifican el pronóstico.

Se reporta un caso de NET por uso de lamotrigina asociado a reciente interrupción de divalproato de sodio, toda vez que los fármacos "anticomiciales" son bien reconocidos como agentes causales: carbamazepina, oxcarbazepina, fenobarbital, lamotrigina y fenitoína, entre otros.

\section{Caso clínico}

Paciente de 68 años de edad con diagnóstico de hipertensión arterial, psoriasis, tabaquismo y enfermedad bipolar en tratamiento fuera del ámbito de nuestra institución, que ingresó a terapia intensiva por extenso rash eritematoso en tronco y extremidades asociado a síndrome febril e hipotensión. Había consultado 15 días antes al servicio de guardia por mareos, fiebre, mialgias y tos, por lo cual fue medicado con amoxicilina-clavulánico que debió suspender a la semana de su inicio por constatar lesiones máculo-eritematosas en tronco y extremidades, interpretadas como reacción alérgica al antibiótico. Tres semanas previas a su ingreso se había modificado su esquema de psicofármacos, habiéndosele indicado lamotrigina $150 \mathrm{mg} /$ día, quetiapina $450 \mathrm{mg}$ /día y clonazepam $2 \mathrm{mg} /$ día.

Las lesiones cutáneas progresaron en extensión y profundidad: se observaba piel eritematosa en forma generalizada con extensas áreas de descamación y flictenas a predominio en miembros inferiores, máculas eritemato purpúricas a nivel cefálico y en extremidades con signo de Nikolsky positivo, áreas de piel denudada en colgajos, erosiones en mucosa genital y oral; edema bipalpebral, conjuntivitis bilateral y pseudomembrana con secreción purulenta (ver figura1).

Durante la evaluación, los familiares refirieron el cambio de medicación habitual de divalproato a lamotrigina con aumento de dosis en dos pasos: de 25 a $50 \mathrm{mg}$ y luego de 50 a $150 \mathrm{mg} /$ día en las 3 semanas previas al inicio de los síntomas. Se suspendió dicha medicación, se inició fluidoterapia, corticoides endovenosos, tratamiento de las lesiones cutáneas con sulfadiazina de plata y analgésicos. Se realizó biopsia de piel que evidenció necrosis epidérmica, despegamiento basal y edema de la dermis superior asociado a infiltrado mononuclear perivascular (ver figura 2).

El paciente evolucionó con hipotensión, taquicardia, taquipnea, fiebre, insuficiencia renal y acidosis metabólica con lactacidemia. Requirió soporte vasopresor con dosis crecientes y asistencia respiratoria mecánica, evolucionando en forma tórpida sin lograr mejoría de las lesiones cutáneas. La familia solicitó adecuación del esfuerzo terapéutico cuando fue evidente que el pronóstico era reservado y sin perspectivas de recuperación. El óbito ocurrió a pesar de las medidas terapéuticas a los 9 días de su ingreso.

\section{Figura I.}

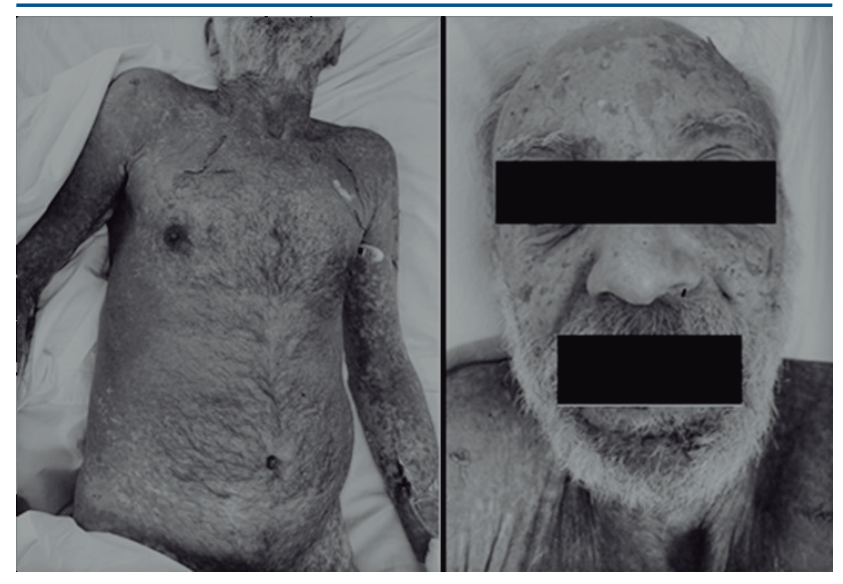

Figura 2.

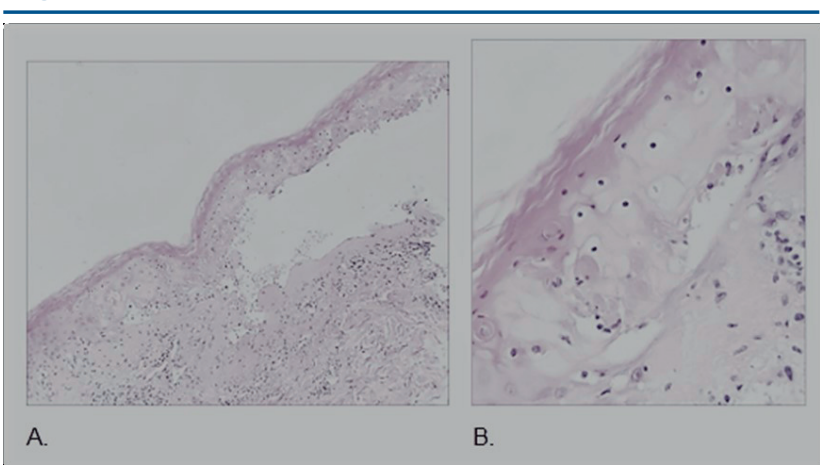

A. H\&E, 100x. Despegamiento dermo-epidérmico.

B. H\&E, 400x. Necrosis epidérmica.

\section{Discusión}

El síndrome de Stevens-Johnson (SSJ) fue descrito por primera vez en 1922 y el término necrólisis epidérmica tóxica fue acuñado por A. Lyell (1956). Tanto el SSJ como el NET representan diferentes grados del mismo tipo de reacción de hipersensibilidad tardía cutánea, difieren por el porcentaje de superficie de piel desprendida o potencialmente desprendible (signo de 
Nikolsky), siendo para SSJ < 15\%, SSJ/NET 15-30\% y para el NET $>30 \%$ (Assier et al., 1995). Se asocia frecuentemente a fármacos entre los que se destacan los anticonvulsivantes (difenilhidantoína, carbamazepina, lamotrigina), antiinflamatorios no esteroideos y antibióticos (sulfamidas, aminopenicilinas, quinolonas y cefalosporinas); algunas infecciones (virus del herpes simple y Micoplasma) y factores genéticos pueden actuar como cofactor precipitante (Cheung et al., 2013; Chung et al., 2004). En el 20\% de los casos no es posible identificar una etiología.

La enfermedad comienza dentro de las 4 semanas de la ingesta del fármaco causal (Dodiuk-Gad et al., 2015). En una revisión sistemática de reacciones adversas cutáneas vinculadas a la lamotrigina, Xiangqing Wang y cols. (2015) evidenciaron que a pesar de haberse reducido la incidencia de $1 \%$ a $0.1-0.01 \%$ con la implementación del incremento gradual y escalonado de dosis cada 15 días, todavía sigue habiendo incrementos de dosis no escalonados; los principales factores de riesgo son la titulación rápida y el uso concomitante con inhibidores de la metabolización y/o glucuronidación de metabolitos de fármacos. En dicha revisión, el $50 \%$ de los pacientes recibió divalproato de sodio asociado y las únicas 3 muertes ocurrieron en dicho grupo (Dodiuk-Gad et al., 2015; Wang et al., 2015).

Los tres elementos claves en el diagnóstico de esta enfermedad son la afectación cutáneo-mucosa (lesiones target atípicas), el compromiso sistémico y el hallazgo histológico.

EL SSJ/NET evoluciona en tres fases sucesivas:

1. Fase prodrómica: se caracteriza por síntomas inespecíficos similares a la gripe y reacciones dermatológicas: malestar general, fiebre, astenia, lesiones maculo-eritematosas que involucran tórax y región proximal de miembros. En esta fase ocurre un error muy común que es atribuir las manifestaciones cutáneas a los antibióticos o antipiréticos, lo que retrasa el diagnóstico del verdadero trastorno y carácter idiosincrático de la respuesta inmunológica frente al fármaco causal;

2. Fase de inflamación: implica mayor afectación cutánea con lesiones maculares de bordes poco definidos y centro violáceo ("target atípicas"), ampollas dolorosas con erosiones y destechamiento en piel y mucosas oftálmica, oral y/o genital asociado a afectación sistémica (fiebre, taquicardia, hipotensión). Son frecuentes las complicaciones pulmonares, hipersecreción bronquial y sobreinfección bacteriana por S. Aureus y P. Aeruginosa con nece- sidad de ventilación mecánica en más del 25\% de los pacientes afectados (Prost et al., 2014) y

3. Fase de meseta: etapa de re-epitelización.

Para una rápida evaluación se dispone de la regla de las “5D” (Dodiuk-Gad et al. 2015): diagnóstico, exposición a drogas, diagnóstico diferencial, determinar causalidad y determinar la gravedad, que permite una sencilla y veloz aproximación inicial. El pronóstico y grado de compromiso puede establecerse identificando 7 factores de riesgo independientes incluidos en la escala de gravedad SCORTEN. Si es positivo para cada uno de los factores clínicos o de laboratorio enumerados (mayor a 40 años, frecuencia cardíaca mayor de 120 latidos/min, urea mayor de $10 \mathrm{mMol} / \mathrm{l}$, glucemia mayor de $14 \mathrm{mMol} / \mathrm{l}$, bicarbonato menor de $20 \mathrm{mMol} / \mathrm{l}$, hemopatía o cáncer activo, afectación muco-cutánea más de $10 \%$ ) suma 1 punto por cado uno de ellos. Debe realizarse los días 1 y 3 del ingreso (Bastuji-Garin et al., 2000; Cartotto et al., 2008).

En el caso que nos ocupa, en el primer día de internación se registró un puntaje de 4 equivalente a una mortalidad del $64 \%$.

Es destacable la histología como uno de los pilares diagnósticos, no fue la excepción en nuestro caso.

La atención de los pacientes debe desarrollarse en Unidades de Terapia Intensiva (UTI) o de quemados y en forma interdisciplinaria. El tratamiento se limita al reconocimiento precoz de los síntomas, identificación y retirada del medicamento implicado, cuidado local de las lesiones, medidas de soporte vital y vigilancia de infecciones sobreagregadas. García-Doval y cols. (2000) en un estudio observacional de 10 años que incluyó a 113 pacientes con NET o SSJ evidenciaron que cuanto antes se retira el fármaco causal, mejor es el pronóstico y la sobrevida (Garcia-Doval et al., 2000). Ningún tratamiento médico específico, como corticoides sistémicos, inmunoglobulinas, ciclosporina e inhibidores de TGF-a, demostró disminuir la mortalidad en ensayos clínicos aleatorizados. La evidencia del uso surge de estudios observacionales retrospectivos cuyos hallazgos son disímiles (Bachot et al., 2003; Kirchhof et al., 2014; Schneck et al., 2008).

En nuestro reporte, el paciente había recibido lamotrigina con un incremento rápido de dosis, asociado a la suspensión reciente de divalproato de sodio y a un diagnóstico previo de psoriasis, que por sí misma podría constituir un mal predictor. Tuvo un periodo prodrómico inicialmente tratado con amoxicilina-clavulánico y luego de la reacción cutánea inicial y ya retirado el antibiótico, se entendió a la lamotrigina como agen- 
te causal. Evolucionó con grave afectación sistémica y posterior neumonía con fallo respiratorio, requiriendo asistencia respiratoria mecánica en forma precoz.

El SSJ/NET ocasiona reacciones cutáneas severas con tasas de mortalidad que pueden superar el 30\%. Si bien la incidencia de la afectación relacionada con lamotrigina ha disminuido en los últimos 20 años gracias a su titulación gradual y progresiva (FDA Briefing Document - US Food and Drug Administration, 2021), es necesario extremar el monitoreo y evitar asociaciones con inhibidores de la metabolización hepática microsomal y de la enzima glucuronil transferasa. La rápida sospecha y el diagnóstico precoz llevan a la principal intervención terapéutica: la inmediata retirada del fármaco responsable, siendo que no existe tratamiento médico específico de comprobada eficacia que modifique sustancialmente la tasa de mortalidad.

Conflictos de intereses: los autores declaran no tener conflicto de intereses.

\section{Referencias bibliográficas}

Assier, H., Bastuji-Garin, S. Revuz, J., \& Roujeau, J.C. (1995). Erythema multiforme with mucous membrane involvement and Stevens-Johnson syndrome are clinically different disorders with distinct causes. Arch Dermatol, 131(5), 539-43.

Bachot, N., Revuz, J., Roujeau, J.C. (2003). Intravenous immunoglobulin treatment for Stevens-Johnson syndrome and toxic epidermal necrolysis: a prospective noncomparative study showing no benefit on mortality or progression. Arch Dermatol, 139(1):33-6. doi: 10.1001/archderm.139.1.33

Bastuji-Garin, S., Fouchard, N., Bertocchi, M., Roujeau, J. C., Revuz, J., \& Wolkenstein, P. (2000). SCORTEN: a severity-of-illness score for toxic epidermal necrolysis. J Investig Dermatol, 115(2), 149-53. doi: 10.1046/j.15231747.2000.00061.x

Cartotto, R., Mayich, M, Nickerson, D., \& Gómez, M. (2008). SCORTEN accurately predicts mortality among toxic epidermal necrolysis patients treated in a burn center. J Burn Care Res, 29(1):141-6. doi: 10.1097/BCR. 0b013e31815f3865
Cheung, Y. K., Cheng, S.H., Chan, E.J., Lo, S.V., Ng, M. H. L., \& Kwan, P. (2013). HLA-B alleles associated with severe cutaneous reactions to antiepileptic drugs in Han Chinese. Epilepsia, 54(7), 1307-14. doi: 10.1111/ epi. 12217

Chung, W.H., Hung, S.I., Hong, H.S., Hsih, M. S., Yang L. Ch., Ho, H. CH., Wu J. Y., \& Chen, Y.T. (2004). Medical genetics: a marker for Stevens-Johnson syndrome. Nature, 428(6982), 486. doi: 10.1038/428486a

Dodiuk-Gad, R. P., Chung, W. H., Valeyrie-Allanore, L., Shear, N. H. (2015). Stevens-Johnson Syndrome and Toxic Epidermal Necrolysis: An Update Am J Clin Dermatol, 16(6), 475-93. doi: 10.1007/s40257-015-0158-0

FDA Briefing Document - US Food and Drug Administration https:// www.fda.gov/media/79324/download. Último acceso: 1 de marzo de 2021.

Garcia-Doval, I., LeCleach, L., Bocquet, H., Otero, X. L., \& Roujeau, J. C. (2000). Toxic epidermal necrolysis and Stevens-Johnson syndrome: does early withdrawal of causative drugs decrease the risk of death? Arch Dermatol, 136(3), 323-7. doi: 10.1001/archderm.136.3.323

Kirchhof, M.G., Miliszewski, M.A., Sikora, S., Papp A., \& Dutz J. P. (2014). Retrospective review of Stevens-Johnson syndrome/toxic epidermal necrolysis treatment comparing intravenous immunoglobulin with cyclosporine. J Am Acad Dermatol, 71(5), 941-7. doi: 10.1016/j.jaad.2014.07.016 Knowles, S., Shear, N. H. (2009). Clinical risk management of StevensJohnson syndrome/toxic epidermal necrolysis spectrum. Dermatol Ther, 22(5), 441-51. doi: 10.1111/j.1529-8019.2009.01260.x

Lyell, A. (1956). Toxic epidermal necrolysis: an eruption resembling scalding of the skin. Br J Dermatol, 68(11), 355-6. doi: 10.1111/j.13652133.1956.tb12766.x

Prost, N., Mekontso-Dessap, A., Valeyrie-Allanore, L., Tran Van Nhieu J., Duong, T-A., Chosidow, O., Wolkenstein, P., Brun-Buisson, Ch., \& Maître, B. (2014). Acute respiratory failure in patients with toxic epidermal necrolysis: clinical features and factors associated with mechanical ventilation. Crit Care Med, 42(1):118-28. doi: 10.1097/CCM.0b013e31829eb94f

Schneck, J., Fagot, J.P., Sekula, P., Sassolas, B., Roujeau, J.C., \& Mockenhaupt, M. (2008). Effects of treatments on the mortality of Stevens-Johnson syndrome and toxic epidermal necrolysis: a retrospective study on patients included in the prospective EuroSCAR study. J Am Acad Dermatol, 58(1), 33-40. doi: 10.1016/i.jaad.2007.08.039

Wang, X., lv, B., Wang H., Zhang, X., Yu, S., Huang X., Zhang J., Tian Ch., \& Lang S. (2015). Lamotrigine-induced severe cutaneous adverse reaction: Update data from 1999-2014. J Clin Neurosci, 22(6), 1005-11. doi: $\underline{10.1016 / \text { i.jocn.2015.01.016 }}$ 